\title{
Isolations of aerobic sporing bacilli from the tips of indwelling intravascular catheters
}

\author{
R. FREEMAN AND B. KING \\ From the Department of Microbiology, University of Leeds
}

SYNOPSIS Following previous reports of the isolation of aerobic sporing bacilli from intravenous $\frac{9}{0}$ catheters and infusion sets, it was decided to analyse a series of catheter tip cultures to ascertain the i significance of these isolations. Those catheters from which aerobic sporing bacilli were isolated ${ }^{\infty}$ behaved statistically differently from other catheters from which organisms were isolated, but were $\vec{f}$ statistically similar to sterile catheters. Using the nitroblue tetrazolium (NBT) test no significant 0 difference was found between patients with isolates of aerobic sporing bacilli and those with sterile $\stackrel{\circ}{工}$ catheters. The NBT test readily distinguished those patients from whom other organisms were $\overrightarrow{7}$ isolated. In a small number of instances some evidence of a possible pathogenic role for the aerobic $\frac{\mathbb{D}}{\mathscr{C}}$ sporing bacillus was found, but the results clearly indicate that in most cases these organisms can be regarded as contaminants.

Several recent reports concerned with the isolation of organisms from the tips of indwelling intravenous catheters (Freeman and King, 1972a; Freeman, King, and Hambling, 1973) and infusion sets (Cox, 1973) have numbered aerobic sporing bacilli amongst the organisms isolated. Aerobic sporing bacilli are almost always regarded as contaminants by microbiologists, and only in exceptional circumstances have they even been considered as human pathogens.

Having recently reviewed our isolations from intravenous and intraarterial catheters, we have examined further those instances in which aerobic sporing bacilli were isolated in an attempt to show whether these isolations are significant.

\section{Methods}

All the patients concerned were subjected to openheart surgery, in whom intravenous catheters are inserted as a routine procedure. The catheter tips were removed under aseptic precautions and cultured as previously described (Freeman et al, 1973). Organisms isolated were identified by routine methods. The whole series will be reported elsewhere but for the purposes of this report three groups of 22 patients have been taken.

GROUP 1

This consists of 22 patients in whom aerobic sporing Received for publication 5 September 1974. bacilli were isolated from the catheter tips. No other infection was present at the time.

GROUP 2

Twenty-two patients in whom organisms other than aerobic sporing bacilli were isolated. The other $\overline{\bar{o}}$ organisms included micrococci, staphylococci, "coliform bacilli', and yeasts. Again, no other infection was present at the time.

\section{GROUP 3}

Twenty-two patients in whom all removed catheter tips were sterile and in whom no other infection was present.

The absence of coexisting infections was shown by daily chest radiographs, white cell count, and culture of sputum, blood, and urine. Specimens for culture were also sent from any other sites, eg, wounds, if clinically indicated.

All patients had a nitroblue tetrazolium (NBT) test performed on the day when the catheter was removed. The test was performed by the method of Freeman and King (1972b).

Finally, the whole series, consisting of 628 catheter tip cultures from 208 patients, was divided $\stackrel{\mathscr{P}}{?}$ into three groups: (A) isolations of aerobic sporing bacilli; (B) isolations of organisms other than aerobic sporing bacilli; (C) sterile catheter tips.

Statistical analysis was performed comparing the data as follows: comparison of mean time in situ for 
groups $\mathrm{A}, \mathrm{B}$, and $\mathrm{C}$ by a rank number series test, deriving the Mann-Whitney $U$ value and so obtaining $\mathrm{p}$; comparison of the mean NBT scores of the three groups of 22 patients (groups 1, 2, and 3) by Student's t test.

\section{Discussion}

Table I shows that the NBT scores in the patients from whom aerobic sporing bacilli were isolated are, in general, within the normal range (1-11\%). If the NBT test is accepted as indicating the presence of bacterial infection in this situation (Freeman and King, 1972), most of these instances would represent contamination. This is supported statistically, since when the mean NBT scores of the sterile catheters and the isolations of aerobic sporing bacilli are compared, $\mathrm{P}<0.025>0.0125$, whereas when comparing these isolations with those of other organisms of more certain pathogenicity $P<0.0005$. Comparison of the isolations of organisms other than aerobic sporing bacilli with the sterile group shows that a clear-cut difference exists $(P<0.0005)$, and confirms our original assertion that the NBT test provides an excellent marker for the presence of organisms in indwelling intravenous catheters, provided that other infection has been excluded (Freeman and King, 1972a).

Before leaving this aspect it is, however, interesting to note that there is some statistical difference between the mean NBT scores for the sterile catheters and the isolations of aerobic sporing bacilli. The possibility that some of these isolations, almost certainly the minority, are significant cannot be ignored. Thus, in three patients aerobic sporing bacilli were isolated from more than one site, and in patient 18 in the aerobic sporing bacilli group the organisms were isolated from a blood culture. The patient concerned received no specific treatment, however, and seemed to have no sequelae. It is notable that the NBT score was elevated in this case.

Turning to table II, statistical analysis shows that there is a highly significant difference between the mean time in situ of the sterile catheters and those

\begin{tabular}{llll}
\hline Days in Situ & $\begin{array}{l}\text { Aerobic Sporing } \\
\text { Bacilli Isolations }\end{array}$ & Other Organisms & Sterile \\
\hline 1 & 18 & 8 & 230 \\
2 & 18 & 17 & 211 \\
3 & 6 & 5 & 61 \\
4 & 1 & 6 & 19 \\
5 & 2 & 4 & 8 \\
6 & 0 & 2 & 1 \\
7 & 0 & 3 & 0 \\
8 & 0 & 2 & 0 \\
9 & 0 & 1 & 0 \\
10 & 0 & 1 & 0 \\
15 & 0 & 2 & 1.8 days \\
Mean time in situ & 1.9 days & 3.89 days & \\
\hline
\end{tabular}

Table II Comparison of days in situ and day of removal of catheters yielding aerobic sporing bacilli, organisms other than $A S B$, and no organisms

\begin{tabular}{|c|c|c|c|c|c|}
\hline \multicolumn{2}{|c|}{ Aerobic Sporing Bacilli Isolations } & \multicolumn{2}{|c|}{ Isolations of 'Coliforms', Staphylococci, etc } & \multicolumn{2}{|c|}{ Sterile Catheters } \\
\hline Patient & NBT Score (\%) & Patient & NBT Score (\%) & Patient & NBT Score $(\%)$ \\
\hline 1 & 11 & 1 & 17 & 1 & 4 \\
\hline 2 & 3 & 2 & 9 & 2 & 5 \\
\hline 3 & 8 & 3 & 21 & 3 & 8 \\
\hline 4 & 11 & 4 & 23 & 4 & 7 \\
\hline 5 & 13 & 5 & 12 & 5 & 11 \\
\hline 6 & 4 & 6 & 16 & 6 & 8 \\
\hline 7 & 8 & 7 & 19 & 7 & 13 \\
\hline 8 & 13 & 8 & 14 & 8 & 5 \\
\hline 9 & 8 & 9 & 18 & 9 & 5 \\
\hline 10 & 13 & 10 & 18 & 10 & 7 \\
\hline 11 & 5 & 11 & 14 & 11 & 8 \\
\hline 12 & 6 & 12 & 12 & 12 & 6 \\
\hline 13 & 17 & 13 & 16 & 13 & 9 \\
\hline $14^{1}$ & 14 & 14 & 20 & 14 & 5 \\
\hline 15 & 6 & 15 & 16 & 15 & 8 \\
\hline 16 & 14 & 16 & 24 & 16 & 4 \\
\hline 17 & 8 & 17 & 5 & 17 & 8 \\
\hline $18^{1}$ & 16 & 18 & 12 & 18 & 9 \\
\hline 19 & 4 & 19 & 16 & 19 & 5 \\
\hline 20 & 6 & $20^{1}$ & 13 & 20 & 8 \\
\hline $21^{1}$ & 2 & 21 & 15 & 21 & 6 \\
\hline & 10 & 22 & 14 & 22 & 5 \\
\hline Mean NBT & $9 \cdot 2$ & Mean NBT & $15 \cdot 6$ & Mean NBT & 7 \\
\hline
\end{tabular}

Table I Comparison of individual and mean NBT scores in three groups of 22 patients none with any known infection other than that shown by catheter tip culture

'Indicates that these patients had the relevant organism isolated from more than one catheter at different sites. 
catheters from which organisms other than aerobic sporing bacilli were isolated $(\mathrm{P}<0.00003)$. However, whilst there is a significant difference between the mean time in situ of the catheters from which aerobic sporing bacilli were isolated and those from which other organisms were isolated $(P<0.06)$, this difference is not found when comparing the mean time in situ of the isolations of aerobic sporing bacilli with the sterile catheters $(P>0 \cdot 3$ ).

In conclusion, our data show that the isolation of aerobic sporing bacilli from the tips of indwelling intravenous catheters, although a common occurrence, is probably of little significance. Care must be taken, however, to judge each case on its merits and an extremely useful guide in this situation is the NBT score for the patient.

It would be unwise on this evidence to include infection of intravenous catheters in the short list of conditions in which aerobic sporing bacilli have been taken to be pathogens (Pearson, 1970).

We thank Mr M. I. Ionescu for encouragement and for his permission to report work carried out on his patients. We also express our gratitude to Dr F. C. Odds for statistical advice.

\section{References}

Cox, G. E. (1973). Bacterial contamination of drip sets. New $Z$. med. $J ., 77,390-392$.

Freeman, R., and King, B. (1972a). Infective complications of indwelling intravenous catheters and the monitoring of infections by the nitroblue-tetrazolium test. Lancet, 1, 992-993.

Freeman, R., and King, B. (1972b). Technique for the performance of the nitroblue tetrazolium (NBT) test. J. clin. Path., 25, 912-914.

Freeman, R., King, B., and Hambling, M. H. (1973). Infective complications of open-heart surgery and the monitoring of infections by the NBT test. Thorax, 28, 617-621.

Pearson, H. E. (1970). Human infections caused by organisms of the bacillus species. Amer. J. clin. Path., 53, 506-515. 